\title{
Expression of AKR1C3 Protein in Human Keloid Skin Tissue
}

\author{
Doo Hyun Nam ${ }^{1}$, DaWoon Lee ${ }^{2}$, \\ Chul Han Kim², Sang Gue Kang ${ }^{2}$, \\ Ho Seong Shin ${ }^{3}$, Young Man Lee ${ }^{1}$ \\ ${ }^{1}$ Department of Plastic and \\ Reconstructive Surgery, Soonchunhyang \\ University College of Medicine, Cheonan; \\ ${ }^{2}$ Department of Plastic and \\ Reconstructive Surgery, College of \\ Medicine, Soonchunhyang University, \\ Seoul; ${ }^{3}$ Department of Plastic and \\ Reconstructive Surgery, College of \\ Medicine, Soonchunhyang University, \\ Bucheon, Korea
}

This work was supported in part by the Soonchunhyang University Research Fund.

No potential conflict of interest relevant to this article was reported.

\begin{abstract}
Background Keloids are abnormal wound responses that are caused by hyperproliferative growth of connective tissue during the healing process. Recent research findings introduced the roles of reactive oxygen species (ROS) in the process of keloid formation. ROS induces oxidative stress and promotes the activities of oxidative damage-inducible genes. Aldo-keto reductase ${ }_{1} C_{3}$ (AKR1C3) prevents destructive ROS toxicity by detoxification of reactive carbonyl species. Thus, this study aimed to compare the expression of $\mathrm{AKR} 1 \mathrm{C}_{3}$ in both normal and keloid skin in vivo.

Methods Six specimens of normal skin and six specimens of keloid tissues from human subjects were used to evaluate the expression of $A K R_{1} C_{3}$ by immunofluorescent staining of tissues and western blotting.

Results By western blotting, it was confirmed that the amount of $A K R_{1} C_{3}$ protein is significantly reduced in keloid tissues compared to normal tissues. Weak expression of AKR1C3 was also found in keloid tissues by immunofluorescent staining.

Conclusions This study confirmed that the expression of AKR1C3 protein participates in ROS metabolism and plays a part in the downregulation of human keloid formation. To the best of our knowledge, this is the first work that reveals that AKR1C 3 can affect the formation of keloids.
\end{abstract}

Keywords Keloid, Reactive oxygen species, Skin

\section{INTRODUCTION}

Keloids are among the most noticeable types of scars and may occur as a result of abnormal wound healing. They are formed by deposition of extracellular matrix components and proliferation of fibroblasts [1]. Keloids compose a pathologic condition characterized by the presence of hyalinized collagen bundles and the existence of chronic inflammatory reactions, such as oxidative stress [2]. Keloid scars extend beyond the margin of the original wound compared with hypertrophic scars. Keloids are estimated to occur

Received: Nov 6, 2015 Revised: Dec 9, 2015 Accepted: Dec 15, 2015 Correspondence: Young Man Lee Department of Plastic and Reconstructive Surgery, Soonchunhyang University College of Medicine, 31 Suncheonhyang 6-gil, Dongnam-gu, Cheonan 31151, Korea. E-mail: youngman@schmc.ac.kr Copyright @ 2016 The Korean Society for Aesthetic Plastic Surgery.

This is an Open Access article distributed under the terms of the Creative Commons Attribution Non-Commercial License (http://creativecommons.org/licenses/by-nc/3.0/) which permits unrestricted non-commercial use, distribution, and reproduction in any medium, provided the original work is properly cited. $\quad$ www.e-aaps.org in about $10 \%$ of people [3] and are seen more often in people with highly pigmented skin such as Black and Asian people rather than Caucasian people [4]. The pathophysiological basis of keloids is yet to be completely determined.

There have been many ongoing keloid-related studies because keloids cause serious functional and aesthetic problems. However, there are no uniformly successful treatments for keloid scars. Excision and primary repair frequently result in recurrence.

It was recently reported that keloid formation is associated with reactive oxygen species (ROS) [3]. According to De Felice et al. [3], significant increases in ROS in keloid tissue fibroblasts are related to oxidative injury and inflammatory reaction.

ROS, including superoxide anion $\left(\mathrm{O}^{2-}\right)$, hydrogen peroxide $\left(\mathrm{H}_{2} \mathrm{O}_{2}\right)$, and hydroxyl anion $\left(\mathrm{OH}^{-}\right)$, can oxidize and modify intracellular molecules. ROS production is induced either directly by oxidants/ oxidases or by a decrease in antioxidants and related upregulation of oxidative damage-inducible genes [2,5]. ROS can directly destroy both DNA and proteins. ROS play a crucial role in the apoptosis of cells injured by oxidation, especially in inflammatory cells 
[6]. Proteins are also important targets for oxidative stress. Recently, it was reported that ROS can act as modulators that regulate signal transduction pathways in various cells [7]. Although human cells have multiple defense systems against free radicals, those that escape such systems can attack and modify subcellular components, including proteins, lipids, and nucleic acids [8].

Lee et al. [9] used OxyBlot analysis to detect proteins damaged by oxidation after 2,4-dinitrophenylhydrazine (DNP) derivatization of carbonyl groups. The authors revealed that proteins stressed by oxidation related to ROS were significantly more highly expressed in keloid tissues compared to normal skin tissue [9].

Aldo-keto reductases (AKRs) are engaged in metabolizing aldehydes that are byproducts of oxidative stress. AKRs are known to generate nicotinamide adenine dinucleotide phosphate (NAPDH)dependent enzymes from several oxidoreductase families and reduce carbonyl groups and ketones of proteins [10]. AKRs are involved in the synthesis of many detoxification compounds [11]. Human AKR1C3 is a stem detoxification enzyme from the AKR superfamily. The physiological functions of AKR1C remain unclear. However, AKR1C3 plays a key role in catalyzing the reductive detoxification of reactive carbonyl species (RCS) and ketones, which are produced by ROS-induced lipid peroxidation reactions [12]. Thus, AKR1C3 prevents destructive ROS toxicity by detoxification of RCS [11].

This study examined the expression of AKR1C 3 in both normal and keloid skin. To determine the correlation between AKR1C3 and keloid formation, the expression of AKR1C3 in normal and keloid skin tissues was analyzed using western blot and immunochemistry.

\section{METHODS}

\section{Tissue samples}

This research protocol, including the use of tissue samples, was reviewed and approved by the Institutional Review Board (IRB) of Seoul Soonchunhyang University Hospital. A total of six normal skin tissue samples and six keloid skin samples were collected from patients who underwent plastic surgery between December 2012 and February 2013 in the Departments of Plastic and Reconstructive Surgery at Soonchunhyang University Hospital in Korea. Six normal skin tissues were obtained from female patients who had breast reconstruction using a latissimus dorsi flap. Six keloid tissues were obtained from patients who had a surgical operation. The obtained tissue specimens were frozen in liquid nitrogen immediately after the resection and kept at $-80^{\circ} \mathrm{C}$ until use. All tissues were used in conventional pathological confirmation and immunohistochemistry for a definite diagnosis. The keloid tissues were fixed with formalin and embedded in paraffin for immunohistochemical studies.

\section{Western blot analysis}

Tissue samples were homogenized in whole-cell enzyme-linked immunosorbent assay (ELISA) buffer. The tissue suspension was rotated for 10 minutes at $4^{\circ} \mathrm{C}$. The supernatants were collected and kept at $-70^{\circ} \mathrm{C}$ until use. Proteins extracted from tissues were separated by sodium dodecyl sulfate polyacrylamide gel electrophoresis (SDS-PAGE) using NuPAGE 4-12\% Bis-Tris gels and transferred to an Immobilon-P membrane. The membrane was blocked with 5\% bovine serum albumin (BSA) in a tris-buffered saline with Tween 20 (TBST) solution, and then reacted with the primary mouse antibody diluted to a 1:1,000 concentration in TBST buffer. The membrane was subsequently washed four times (10 minutes, $10 \mathrm{~min}$ utes, 15 minutes, and 15 minutes) and then stored at $4^{\circ} \mathrm{C}$ for 16 hours. Proteins on the membrane were detected using an enhanced chemiluminescence solution kit. The membranes were stripped and reblotted with anti-actin antibody.

\section{Immunohistochemical staining}

Paraffin sections $(4 \mu \mathrm{m})$ were deparaffinized in xylene, rehydrated in $10 \mathrm{mM}$ citrate buffer ( $\mathrm{pH}$ 6.0), and then heated in a microwave oven for 15 minutes to restore antigens. To suppress endogenous peroxidase within tissues, the tissues were treated with $3 \%$ peroxide for 5 minutes and then with blocking solution for 30 minutes. The slides were then incubated with primary mouse anti-AKR1C3 antibody (catalog \#A6229; Sigma, St. Louis, MO, USA) in a damp environment for 1 hour. Tissues were stained with 3,3'-diaminobenzidine (ScyTek, Logan, UT, USA). The quantitative analysis of the comparative expression between keloid and normal skin tissues as expressed by western blotting was made with the TINA software version $2.10 \mathrm{e}$ (Raytest Isotopenmessgeraete, Straubenhardt, Germany) software program.

\section{Statistical analysis}

The data from the Raytest TINA program were evaluated by SPSS 17.0 (IBM Corp., Armonk, NY, USA). Our team used the nonparametric Mann-Whitney test to compare the keloid and normal skin groups. If a P-value was less than 0.05 , it was considered highly statistically significant.

\section{RESULTS}

\section{Immunohistochemical examination}

In the immunohistochemical study, AKR1C3 was weakly expressed in keloid skin tissues but rather strongly expressed in normal skin tissues (Fig. 1). The same result was observed in all six keloid tissues.

\section{Western blot analysis}

According to western blotting results, AKR1C3 protein was expressed in both keloid and normal skin tissues. However, AKR1C3 pro- 

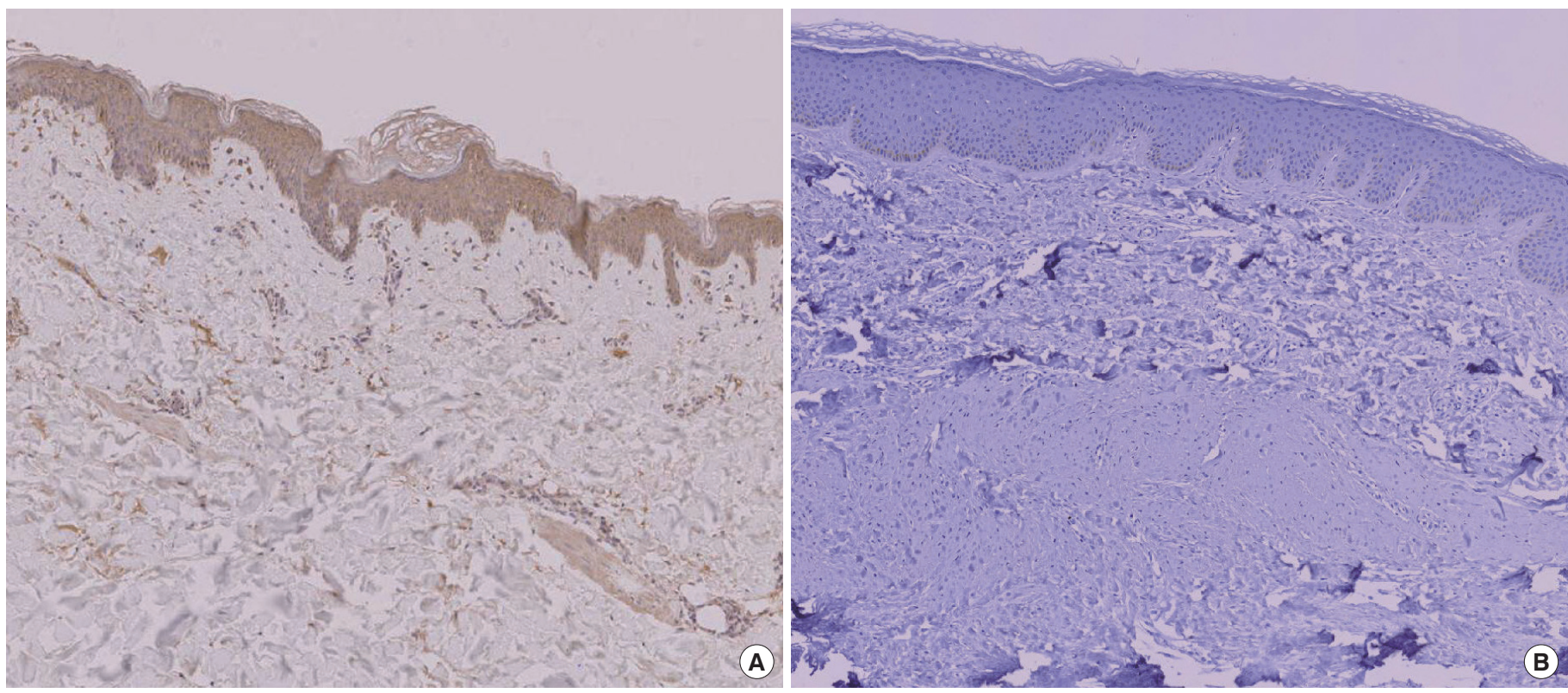

Fig. 1. Immunohistochemical study. (A) Aldo-keto reductase 1C3 (AKR1C3) in normal skin (immunochemical stain, $\times 20)$. (B) AKR1C3 in keloid tissue (Immunochemical stain, $\times 100)$.

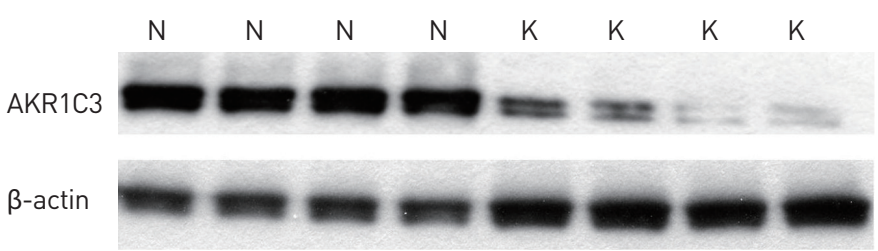

Fig. 2. Western blot analysis. Aldo-keto reductase $1 \mathrm{C} 3$ (AKR1C3) in normal ( $N$ ) and keloid ( $K$, diabetic) skin tissue.

tein was expressed at relatively lower levels in keloid skin tissues compared with normal skin tissues (Fig. 2). The Raytest TINA software program showed that relative protein expression was an average of $1.92(\mathrm{SD}=0.083)$ in normal skin and $0.57(\mathrm{SD}=0.190)$ in keloid skin tissues (Fig. 3). These results indicate significant statistical differences in protein expression between normal and keloid skin tissues according to the Mann-Whitney U-test $(\mathrm{P}<0.05)$. The immunohistochemical results are in good agreement with the western blot results in terms of AKR1C3 expression.

\section{DISCUSSION}

There are several reports that AKR1C 3 is associated with ROS [2, 11]. However, there has been no research on AKR1C3 protein levels and their relation to oxidative damage in keloids. Therefore, we aimed to compare AKR1C3 expression in keloid and normal skin tissues.

In this study, we measured AKR1C3 in keloid and normal skin tissues by western blotting and immunohistochemistry. We found that keloid skin tissues have lower AKR1C3 protein levels than do

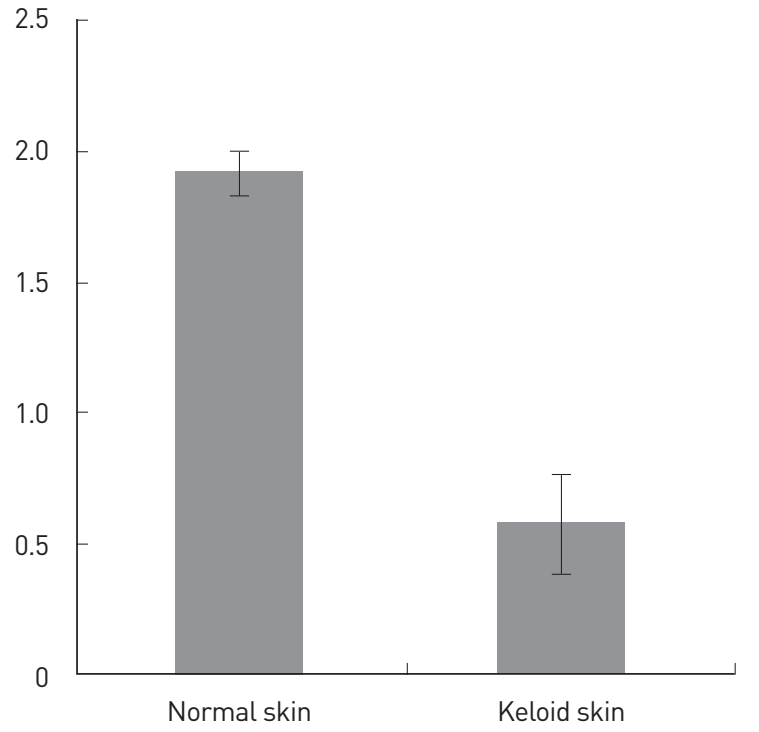

Fig. 3. Relative protein expression of Aldo-keto reductase 1C3 (AKR 1C3). The Raytest TINA software was used to assess protein expression in normal $(S D=0.083)$ and keloid $(S D=0.190)$ skin $(P<0.05)$.

normal skin tissues.

Many biochemical processes are involved in cellular oxidationreduction reactions. Proteins are one of the major targets for oxidative stress [8]. The skin is also a major target for oxidative stress, as it is constantly irradiated by Ultraviolet (UV) light and other environmental stimuli that produce ROS [13]. ROS are produced as a result of metabolic products during chronic inflammatory reactions in keloids, affecting mainly cellular protein, carbohydrate, and lipid 
metabolism.

ROS act as polyunsaturated fatty acids to generate lipid peroxides, known as RCS, including 4-hydroxy-2-nonenal (4-HNE) [11]. ROS can modify the carbonyl groups of amino acids in proteins, including arginine, lysine, threonine, and proline residues [10]. Oxidative reactions may cause an increase in RCS, which promote chemical modifications of proteins and induce tissue damage. RCS damage multiple cellular processes, such as calcium homeostasis, ion channel modulation, and energy production [14-16]. In addition, RCS also can affect the regulation of cell signaling, proliferation, and adaptation to stress. Previous studies found increased levels of ROS in keloids and proposed their implication in the pathogenesis of keloids [17]. Evidence of ROS accumulation supports the hypothesis that oxidative stress is a mechanism of cellular proapoptotic actions in keloid cells [18].

As mentioned above, a previous study reported that RCS are more highly expressed in keloid tissues than in normal skin using the OxyBlot kit [9]. This kit has the advantage of performing a simple and quantitative immunodetection of carbonyl groups [19]. The side chains of carbonyl groups are derivatized to DNP via a reaction with 2,4-DNP. The DNP-derivatized proteins are separated by electrophoresis and analyzed by western blot using an antibody specific to DNP proteins. After incubation with a peroxidase-conjugated secondary antibody, the membranes are then treated with luminol to detect the antigen/primary antibody/secondary antibody/peroxidase complex bound to the membrane. Thus, using this kit, RCS related to oxidatively modified proteins can be detected [9]. Furthermore, the oxidative status of each protein can be analyzed quantitatively by comparison of the signal strength [19].

There are carbonyl-reducing enzymes such as antidiuretic hormone $(\mathrm{ADH})$, aldehyde dehydrogenase (ALDH), glutathione Stransferases (GSTs), and AKRs that lower the level of RCS via detoxification. The AKR superfamily consists of more than 100 members. In humans, 10 enzymes fall in the following subfamilies: AKR1A, AKR1B, AKR1C, AKR1D, and AKR7A, which are expressed in the tissues of the liver, brain, and kidney [20,21].

The AKR superfamily is characterized as monomeric, cytosolic, NADPH-dependent oxidoreductases that convert carbonyl groups into alcohols [22]. AKRs use pyridine nucleotides as coenzymes to catalyze multiple reactions involved in the metabolism of carbohydrates, steroids, glycosylation end products, and RCS. GST is catalyzed 4-hydroxynonenal (4-HNE), forming GST-conjugates. GSTconjugates of 4-HNE are related to reactive aldehydes and ketones [23,24]. 4-HNE can be reduced by AKR1C1 - C4 and AKR1A1, of which AKR1C1 is the best known among them [25,26]. 4-HNE is removed by glutathione (GSH), which is related to GST under normal conditions. At times of GSH depletion, AKR1C1 acts as an effective cellular barrier under conditions of oxidative stress [11].

The physiological functions of AKR1Cs remain unclear, but members of AKR1C3 are considered major players in catalyzing the re- ductive detoxification of RCS and ketones, which are produced as a result of oxidative stress. The decrease in AKR1C3 that occurs under conditions of increased oxidative stress involved in the pathogenesis of keloid formation is one of the defense mechanisms against ROS toxicity [11].

Our study demonstrated that only a small amount of AKR1C3 is expressed in keloids by western blot analysis. Thus, keloid skin tissues may not be able to defend against oxidative stresses, such as ROS. Increased fibroblast proliferation in keloid skin is related to the increase in ROS generation and downregulation of detoxification enzymes such as AKR1C3, which account for the excessive proliferation of keloids. Accordingly, it is concluded that the decreased expression of AKR1C3 is associated with skin keloids in the altered metabolic state of increased ROS generation. Further studies are needed to understand the exact mechanism of signal transduction and modulation of AKR1C3 in keloids.

\section{REFERENCES}

1. Ehrlich HP, Desmouliere A, Diegelmann RF, et al. Morphological and immunochemical differences between keloid and hypertrophic scar. Am J Pathol 1994;145:105-13.

2. Lee YJ, Lee GJ, Baek BJ, et al. Cadmium-induced up-regulation of aldo-keto reductase 1C3 expression in human nasal septum carcinoma RPMI-2650 cells: involvement of reactive oxygen species and phosphatidylinositol 3-kinase/Akt. Environ Toxicol Pharmacol 2011;31: 469-78.

3. De Felice B, Garbi C, Santoriello M, et al. Differential apoptosis markers in human keloids and hypertrophic scars fibroblasts. Mol Cell Biochem 2009;327:191-201.

4. Alhady SM, Sivanantharajah K. Keloids in various races. A review of 175 cases. Plast Reconstr Surg 1969;44:564-6.

5. Dröge W, Schulze-Osthoff K, Mihm S, et al. Functions of glutathione and glutathione disulfide in immunology and immunopathology. FASEB J 1994;8:1131-8.

6. Simon HU, Haj-Yehia A, Levi-Schaffer F. Role of reactive oxygen species (ROS) in apoptosis induction. Apoptosis 2000;5:415-8.

7. Suzuki YJ, Forman HJ, Sevanian A. Oxidants as stimulators of signal transduction. Free Radic Biol Med 1997;22:269-85.

8. Chance B, Sies H, Boveris A. Hydroperoxide metabolism in mammalian organs. Physiol Rev 1979;59:527-605.

9. Lee YJ, Kwon SB, Kim CH, et al. Oxidative Damage and Nuclear Factor Erythroid 2-Related Factor 2 Protein Expression in Normal Skin and Keloid Tissue. Ann Dermatol 2015;27:507-16.

10. Chevion M, Berenshtein E, Stadtman ER. Human studies related to protein oxidation: protein carbonyl content as a marker of damage. Free Radic Res 2000;33 Suppl:S99-108.

11. Barski OA, Tipparaju SM, Bhatnagar A. The aldo-keto reductase superfamily and its role in drug metabolism and detoxification. Drug Metab Rev 2008;40:553-624. 
12. Penning TM, Drury JE. Human aldo-keto reductases: function, gene regulation, and single nucleotide polymorphisms. Arch Biochem Biophys 2007;464:241-50.

13. Bickers DR, Athar M. Oxidative stress in the pathogenesis of skin disease. J Invest Dermatol 2006;126:2565-75.

14. Niki E. Lipid peroxidation: physiological levels and dual biological effects. Free Radic Biol Med 2009;47:469-84.

15. Humphries KM, Yoo Y, Szweda LI. Inhibition of NADH-linked mitochondrial respiration by 4-hydroxy-2-nonenal. Biochemistry 1998;37: 552-7.

16. Bhatnagar A. Electrophysiological effects of 4-hydroxynonenal, an aldehydic product of lipid peroxidation, on isolated rat ventricular myocytes. Circ Res 1995;76:293-304.

17. Corrocher R, Ferrari S, de Gironcoli M, et al. Effect of fish oil supplementation on erythrocyte lipid pattern, malondialdehyde production and glutathione-peroxidase activity in psoriasis. Clin Chim Acta 1989; 179:121-31.

18. Mantel A, Newsome A, Thekkudan T, et al. The role of aldo-keto reductase 1C3 (AKR1C3)-mediated prostaglandin D2 (PGD2) metabolism in keloids. Exp Dermatol 2016;25:38-43.

19. Dalle-Donne I, Rossi R, Giustarini D, et al. Protein carbonyl groups as biomarkers of oxidative stress. Clin Chim Acta 2003;329:23-38.
20. Hyndman D, Bauman DR, Heredia VV, et al. The aldo-keto reductase superfamily homepage. Chem Biol Interact 2003;143-144:621-31.

21. O'Connor T, Ireland LS, Harrison DJ, et al. Major differences exist in the function and tissue-specific expression of human aflatoxin B1 aldehyde reductase and the principal human aldo-keto reductase AKR1 family members. Biochem J 1999;343 Pt 2:487-504.

22. Jin Y, Penning TM. Aldo-keto reductases and bioactivation/detoxication. Annu Rev Pharmacol Toxicol 2007;47:263-92.

23. Ramana KV, Dixit BL, Srivastava S, et al. Selective recognition of glutathiolated aldehydes by aldose reductase. Biochemistry 2000;39:1217280.

24. Srivastava S, Conklin DJ, Liu SQ, et al. Identification of biochemical pathways for the metabolism of oxidized low-density lipoprotein derived aldehyde-4-hydroxy trans-2-nonenal in vascular smooth muscle cells. Atherosclerosis 2001;158:339-50.

25. Khanna M, Qin KN, Wang RW, et al. Substrate specificity, gene structure, and tissue-specific distribution of multiple human 3 alpha-hydroxysteroid dehydrogenases. J Biol Chem 1995;270:20162-8.

26. Burczynski ME, Sridhar GR, Palackal NT, et al. The reactive oxygen species--and Michael acceptor-inducible human aldo-keto reductase AKR1C1 reduces the alpha,beta-unsaturated aldehyde 4-hydroxy-2nonenal to 1,4-dihydroxy-2-nonene. J Biol Chem 2001;276:2890-7. 